“(C) 2018 IEEE. Personal use of this material is permitted. Permission from IEEE must be obtained for all other uses, in any current or future media, including reprinting/republishing this material for advertising or promotional purposes, creating new collective works, for resale or redistribution to servers or lists, or reuse of any copyrighted component of this work in other works." 


\title{
A Dual-Layered Loop Array Antenna for Base Stations With Enhanced Cross-Polarization Discrimination (XPD)
}

\author{
Can Ding, Member, IEEE, Hai-Han Sun, Student Member, IEEE, Richard W. Ziolkowski, Fellow, IEEE \\ and Y. Jay Guo, Fellow, IEEE,
}

\begin{abstract}
This paper presents a novel dual-loop array antenna targeted at current and future base station applications. The antenna has four rectangular loops and four trapezoidal loops printed on the front and back sides, respectively, of a substrate placed above a flat square reflector. All eight loop radiators are excited simultaneously with properly designed feed networks to achieve its $\pm 45^{\circ}$ polarization states. The trapezoidal loops act like folded (electric) dipoles; the rectangular loops act primarily as magnetic dipoles. The combination of these two loop arrays leads to a type of magneto-electric (ME) loop antenna that has stable directivity patterns with high cross-polarization discrimination (XPD) values across a $\mathbf{4 5 . 5 \%}$ operational fractional bandwidth from 1.7 to $2.7 \mathrm{GHz}$. A fabricated and measured prototype confirms the simulation results and demonstrates that the halfpower-beamwidths (HPBWs) in the horizontal plane vary between $63^{\circ}$ to $70^{\circ}$, the XPD value is $>20 \mathrm{~dB}$ in the boresight direction, and is $>10 \mathrm{~dB}$ within the entire cellular coverage angular range: $-60^{\circ} \leq \theta \leq 60^{\circ}$.
\end{abstract}

Index Terms-Antenna array, base station antenna, crosspolarization discrimination (XPD), dual-polarization, loop antenna, magneto-electric antenna, magneto-electric array

\section{INTRODUCTION}

M ODERN commercial base station antenna elements must meet several industry-recognized performance specifications. These specifications include good impedance matching to $50 \Omega$ across a wide frequency range from 1.71 to $2.69 \mathrm{GHz}$, dual-polarizations, high port-to-port isolation, stable half-power-beamwidth (HPBW), and high crosspolarization discrimination (XPD). There have been many antenna research activities in this area in recent years. Generally speaking, the reported antennas can be classified into three different types depending on their configurations and operating principles.

The first one employs cross-dipoles, i.e., the radiating elements are a pair of dipoles that are oriented perpendicular to each other [1-12]. By exciting either one of the two subdipoles, different polarizations can be obtained. The basic cross-dipole configurations used in early base station applications $[1,2]$ had the employed two dipoles "isolated" from

Manuscript submitted 16 Mar. 2018; revised 24 Jun. 2018. This work was funded by Australian Research Council (ARC) DP160102219.

All the authors are with the Global Big Data Technologies Centre (GBDTC), University of Technology Sydney (UTS), Ultimo NSW 2007, Australia. e-mail:(can.ding.1989@gmail.com)

Richard W. Ziolkowski is also with the Department of Electrical and Computer Engineering, The University of Arizona, Tucson, AZ 85721 USA. email: ziolkows@email.arizona.edu.

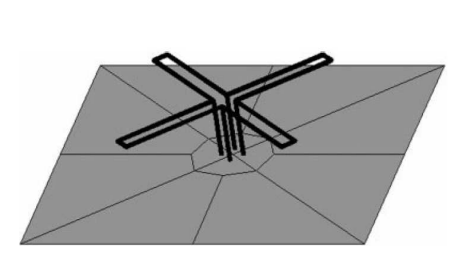

(a)

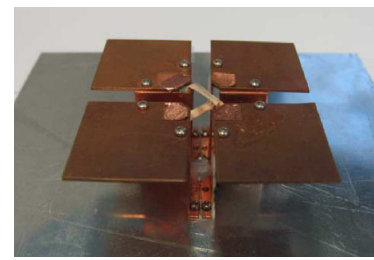

(c)

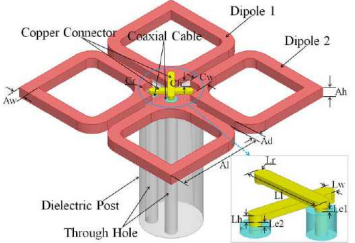

(b)

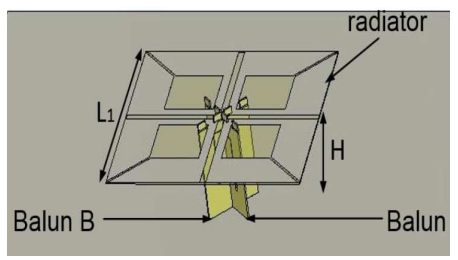

(d)
Fig. 1. Example configurations of different types of base station antennas. (a) Type-I "isolated" cross-dipole [1]. (b) Type-I "tightly-coupled" cross-dipole [5]. (c) Type-II ME dipole [14]. (d) Type-III square array antenna [24].

each other, as shown in Fig. 1(a), resulting in bandwidths that were usually $<25 \%$. This limited bandwidth does not fully cover the $4 \mathrm{G}$ bands. More recently, it has been advocated to exploit cross-dipoles with wider dipoles and to decrease the spacings between them, thus making them more tightly coupled to each other [3-12], as shown in Fig. 1(b). These configurations can have much wider bandwidths and improved radiation performance. Nonetheless, their radiation patterns usually vary significantly with frequency. To achieve stable radiation patterns as required for the base station application, shaped reflectors are usually employed to compensate for the patterns changing with frequency. While these modified reflectors are acceptable in single band base station arrays, they may result in unwanted scattering to other antennas in advanced systems such as the current $4 \mathrm{G}$ dual-band array systems or the anticipated 5G massive MIMO systems [13].

The second type is based on magneto-electric (ME) dipoles [14-19]. ME dipoles consist of a pair of crossed dipoles, a reflector, and a pair of patch antennas which are placed between the cross dipoles and the reflector, see Fig. 1(c). The advantage of the ME dipoles is the stability of their radiation patterns across a wide band, a feature that arises from the complementary electric and magnetic currents generated on the dipole and patch radiators. Their effective electric and magnetic currents are orthogonal to each other, and they are all 
parallel to the ground plane. According to image theory [20], the corresponding radiation patterns vary in opposite ways with frequency, thereby generating stable combined patterns. The drawback of ME dipoles is the challenge to achieve an excellent impedance match, i.e., with VSWR $<1.5$. Most of the published work has moderate matching values, i.e., typically with VSWR $<2$. A recently published ME dipole [19] has achieved excellent matching with VSWR $<1.5$ and a high port-to-port isolation from 1.62 to $2.87 \mathrm{GHz}$. However, it appears that maintaining stable radiation patterns over this bandwidth with this structure is challenging.

The third type, referred to as a square array, utilizes four electric dipoles arranged in a square [13,21-24] as shown in Fig. 1(d). By properly exciting them, dual slant-polarized (i.e, $\pm 45^{\circ}$ polarized) radiation can be achieved. This configuration type normally has a limited bandwidth, i.e., $<25 \%$. However, recently published papers $[13,24]$ have demonstrated a significantly enhanced impedance bandwidth, $>45 \%$, and the ability to maintain a stable radiation pattern over that frequency range.

It should be note that most works have focused only on achieving excellent matching and stable HPBW. Even though a high XPD value has become another important criterion for base station antennas [25,26], only a few systems have been reported that have achieved this goal. The XPD technical standards are $>20 \mathrm{~dB}$ in the boresight direction and $>10 \mathrm{~dB}$ within $\pm 60^{\circ}$ of the main lobe. While it is relatively easy to have the XPD $>20 \mathrm{~dB}$ at boresight, it is a real challenge to maintain the XPD $>10 \mathrm{~dB}$ over the entire coverage range, $-60^{\circ} \leq \theta \leq 60^{\circ}$.

Ref [25] first addressed the XPD issue, attaining good XPD values across a wide band. However, large parasitic elements were employed, which significantly enlarged the antenna aperture. Based on an equivalent model and a comprehensive quantitative analysis of dual-slant-polarized antennas, it has been demonstrated that a large aperture is indeed required to enhance the XPD of a cross-dipole configuration [12]. Nevertheless, in a later paper [26], vertical parasitic elements were placed between the cross-dipole and its reflector to dramatically increase the XPD without increasing the aperture size. Unfortunately, the realized bandwidth was limited to $11.8 \%$. Furthermore, it is noted that these XPD enhancing methods are all limited to cross-dipole type configurations.

In this paper, a novel configuration that employs eight loopbased radiators that leads to stable radiation patterns and high XPD values across a very wide operational band, is reported. In this antenna, a smaller loop array that employs four rectangular loop radiators is printed on the back side of a substrate and a larger loop array that is constructed with four trapezoidal loop radiators is printed on the front side. The antenna reported herein operates with a different mechanism when it is compared with the published ME dipoles that have one pair (single polarization) or two pairs (dual polarization) of electric current and magnetic current perpendicular to each other and parallel to the ground plane [14-18]. The eight radiating elements in the antenna reported support both magnetic and electric currents operating in concert, the magnetic ones being oriented in a direction perpendicular to the electric ones. The electric currents are parallel to the ground plane

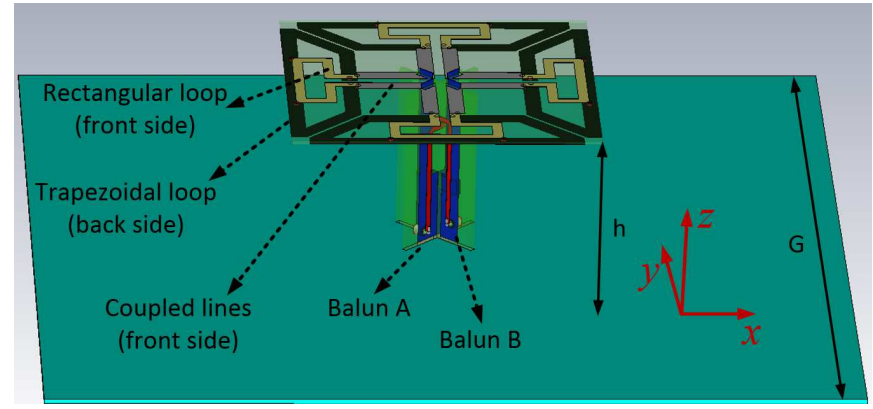

(a)

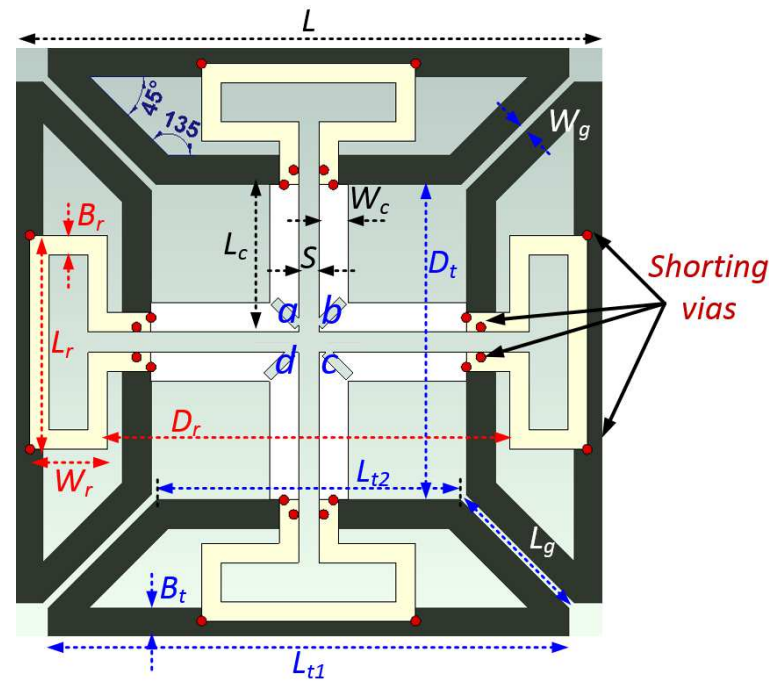

(b)

Fig. 2. Dual-layered loop array antenna. (a) Detailed perspective view. (b) Top view of the radiation aperture.

while the magnetic currents are normal to it. As a result, the fields radiated by the two loop arrays compensate for each other, leading to improved XPD values and stabilized HPBWs across a wide band. The experimental results demonstrate, in good agreement with their simulated values, that the XPD is $>20 \mathrm{~dB}$ at boresight and $>10 \mathrm{~dB}$ within the coverage range, $-60^{\circ} \leq \theta \leq 60^{\circ}$. Impedance matching with $S_{11}<-10 \mathrm{~dB}$ is achieved across a wide operational band, from 1.7 to $2.7 \mathrm{GHz}$, i.e., $1.0 \mathrm{GHz}$, yielding a fractional bandwidth $\mathrm{FBW}=45.45 \%$ for base station applications. The attained HPBWs within this band are from $63^{\circ}$ to $70^{\circ}$.

\section{Antenna Configuration}

Fig. 2(a) gives a detailed perspective view of the duallayered loop array antenna. It has an aperture consisting of eight loop radiators printed on the two sides of a substrate. Two baluns are employed to support these radiators and to achieve their $\pm 45^{\circ}$ polarization states. The antenna is placed on a flat square reflector whose size is $G \times G$. Fig. 2(b) illustrates the details of the radiating elements. A smaller loop array consisting of four rectangular loops is printed on the front side of the substrate; a larger loop array consisting of four trapezoidal loops is printed on the back side. Four pairs of coupled lines used to excite the radiators are also printed on the front side of the substrate. Shorting vias are inserted into 
TABLE I

DESIGN DIMENSIONS (IN MILLIMETRES)

\begin{tabular}{|c|l|l|}
\hline Parameter & Value & Description \\
\hline$L$ & 60.8 & Aperture size \\
\hline$h$ & 33 & Antenna height \\
\hline$G$ & 160 & Reflector size \\
\hline$L_{r}$ & 22 & Length of rectangular loops \\
\hline$W_{r}$ & 8 & Width of rectangular loops \\
\hline$B_{r}$ & 2 & Strip width of rectangular loops \\
\hline$D_{r}$ & 41.55 & Distance between rectangular loops \\
\hline$L_{t 1}$ & 53.77 & Edge length of trapezoidal loops 1 \\
\hline$L_{t 2}$ & 31.5 & Edge length of trapezoidal loops 2 \\
\hline$L_{g}$ & 15.75 & Edge length of trapezoidal loops 3 \\
\hline$W_{g}$ & 0.74 & Gap width between trapezoidal loops \\
\hline$D_{t}$ & 32.55 & Distance between trapezoidal loops \\
\hline$L_{c}$ & 15.23 & Length of coupled lines \\
\hline$W_{c}$ & 3.0 & Width of coupled lines \\
\hline$S$ & 2.1 & Distance between a pair of coupled lines \\
\hline
\end{tabular}

the substrate to connect the loop radiators printed on both of its sides and to facilitate them being excited coherently. These shorting vias also prevent unwanted resonances that might result from the coupling between the radiators in both arrays. The substrate employed in this work is a $0.5 \mathrm{~mm}$ thick piece of FR4. Its relative dielectric constant is 4.3, and its loss tangent is 0.021 . The detailed dimensions of the radiators are given in Fig. 2(b) and their optimized values are given in Table I. The distance between this radiation aperture and the reflector is $h$. A more detailed description of the feed networks will be given in Section IV-B.

\section{Operating PRinciples}

To better understand its working mechanisms, the antenna system was analysed numerically. All of these simulations were conducted in CST Microwave Studio 2017. The analysis first focused on the eight loop radiators in the presence of the ground plane. They were excited simultaneously using four ideal discrete ports as shown in Fig. 3(a). The feed networks, including the coupled lines, were removed in these studies to improve the simulation time. These components were found to have only minor effects on the radiation pattern and were included later in the final optimization studies.

\section{A. Current Distribution}

The current distributions at the center frequency $2.2 \mathrm{GHz}$ on the small and large loop arrays are illustrated in Fig. 3(b) and $3(\mathrm{c})$, respectively. Two current monitors $(\mathrm{C} 1$ and $\mathrm{C} 2)$ were used to sample the current at indicated specific points, i.e. they were applied to the middles of the outer sides of the rectangular and trapezoidal loops as shown in Fig. 3(a). The monitored values are given in Fig. 4. They provided a means to calculate the magnitudes and phases of the currents on the radiators of both loop arrays.

The currents induced on the rectangular loops are illustrated in Fig. 3(b). The stronger components of these currents reside on their inner edges. The currents forming the magnetic dipoles are clearly identifiable. The orientation of each magnetic dipole, as depicted in the right subplot, follows immediately from the direction of the currents in the

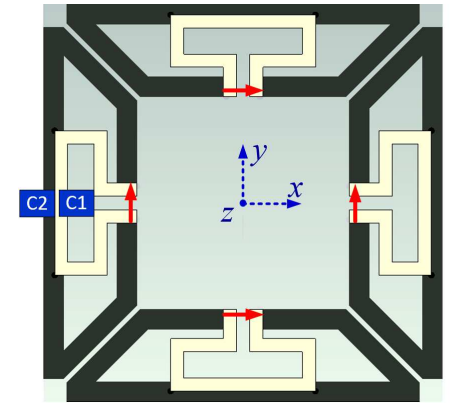

(a)

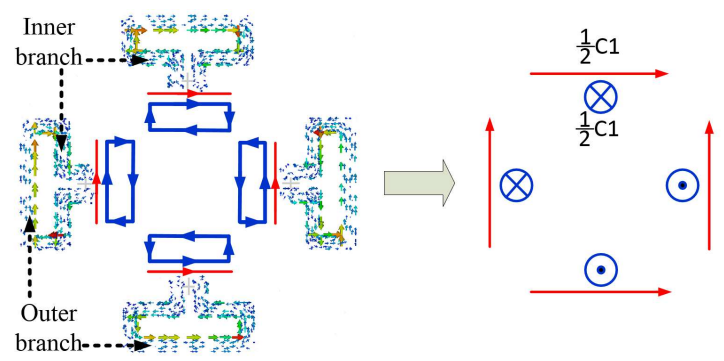

(b)

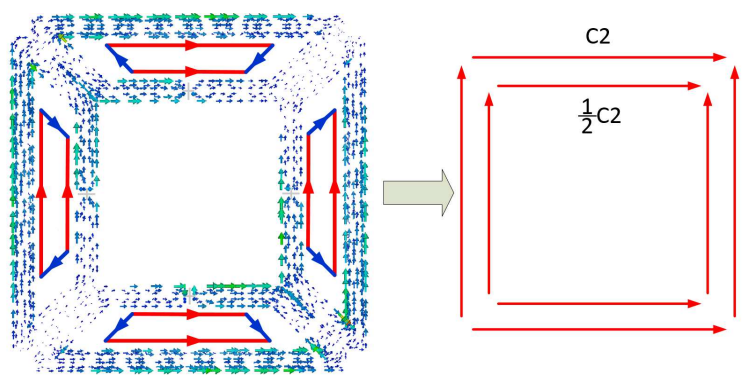

(c)

Fig. 3. Models of the radiating elements. (a) Simplified model with ideal excitations. Surface current distribution on the (b) front side of the small rectangular loops, and (c) back side of the large trapezoidal loops at the center frequency, $2.2 \mathrm{GHz}$.

corresponding loop. The currents on the outer branches of the inner edges can be effectively decomposed into two pieces: a strong electric component and the magnetic component along it that completes the loop current. The electric dipoles are clearly oriented by the current directions along those outside branches, as depicted in the right subplot. As a result, each rectangular loop acts as the magnetic and electric dipole combination illustrated in the right subplot. These features are also emphasized with the red and blue current direction inserts in the left subplot.

The currents on the trapezoidal loops are illustrated in Fig. 3(c). They have a standard folded dipole behavior [20]. They are concentrated on the inner and outer edges of the trapezoids as shown in the left subplot. Moreover, they can be generally considered to be in phase. As a consequence, these currents can be viewed simply as a combination of two linear electric currents as depicted in the right subplot.

By applying more current monitors at different positions on the rectangular and trapezoidal loops, more accurate current density distribution information was attained. It was found that the weighting factors of the various current components can be 


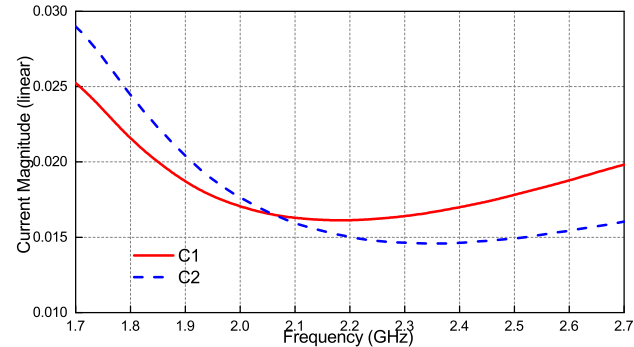

(a)

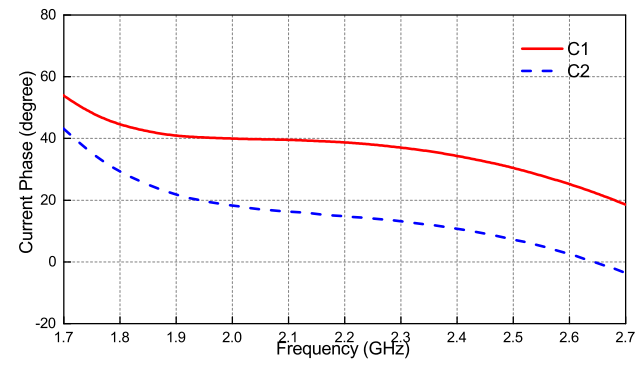

(b)

Fig. 4. The (a) magnitudes and (b) phases of the current monitor C1 and C2.

approximated as $\frac{1}{2} C 1: \frac{1}{2} C 1$ for the rectangular loops and as $C 2: \frac{1}{2} C 2$ for the trapezoidal loops. These factors are explicitly shown in the right subplots of both Figs. 3(b) and 3(c).

Figs. 4(a) and 4(b) show the simulated magnitudes and phases of the currents measured by monitors $\mathrm{C} 1$ and $\mathrm{C} 2$, respectively. It was determined from these values that at the middle of the outer sides of the rectangles and trapezoids, where they reach their peak values, the currents have similar magnitudes, i.e., $|C 1| \approx|C 2|$, and small phase differences, i.e., $<20^{\circ}$. This behavior is expected since the outer sides of the loops are shorted using vias. Note that the electric currents dominate the co-polarization radiation patterns. The magnetic currents only help mitigate the cross-polarization radiation. Since the current densities of the electric current on the larger loops are much stronger $(1.5 \times \mid \mathrm{C} 2 \mathrm{l})$ than those on the smaller loops $(0.5 \times \mid \mathrm{C} 1 \mathrm{l})$, the characteristics of the fields radiated by the system are dominated by them.

\section{B. Radiation Pattern}

To explore the differences between exciting each array separately and both arrays simultaneously, we have plotted their co- and cross-polarization directivity patterns in Figs. 5(a), 5(b), and 5(c), respectively. The simulations were conducted with the ground plane being present. Six frequency samples, $f$ $=1.7,1.9,2.1,2.3,2.5$, and $2.7 \mathrm{GHz}$, were selected across the target band in each case. Moreover, note that the directivity patterns generated by the small and large loop arrays presented in Figs. 5(a) and 5(b), respectively, were obtained by exciting only one array amd removing the other array from simulation.

It is observed from these results that the cross polarization level at $\theta= \pm 60^{\circ}$ is noticeably lower when both arrays are excited together. This outcome was a main goal of this work. Moreover, while the co-polarization patterns shown in Figs. 5(b) and 5(c) are almost identical, they are different from those

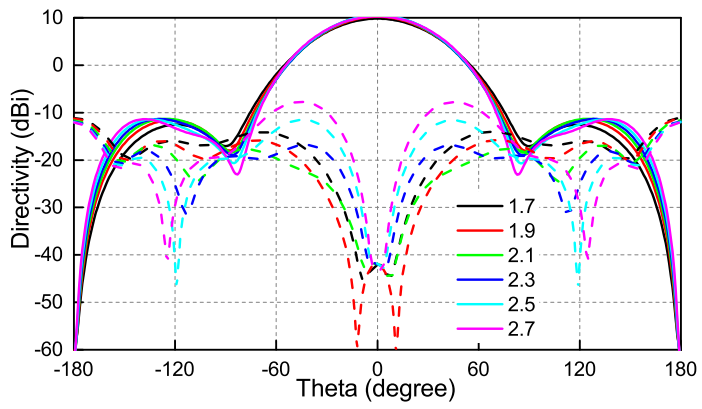

(a)

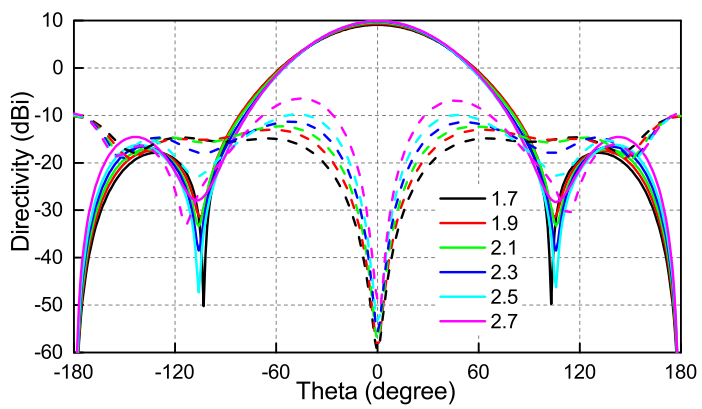

(b)

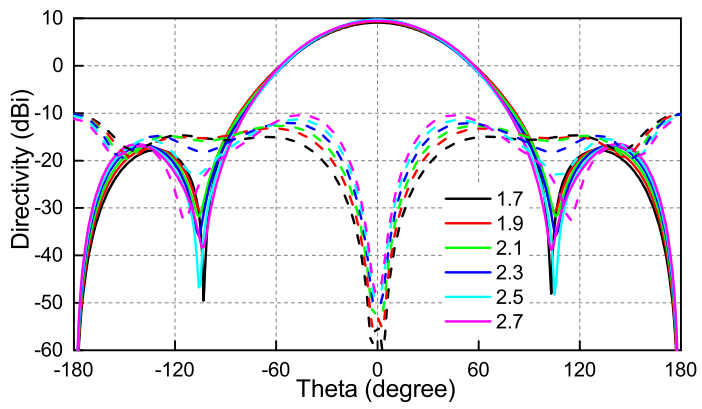

(c)

Fig. 5. Simulated co- and cross-polarization directivity patterns in the horizontal plane at the frequency points: $1.7,1.9,2.1,2.3,2.5$, and $2.7 \mathrm{GHz}$. They are represented, respectively, by the solid and dashed lines. (a) Four small loops only. (b) Four large loops only. (c) All eight loops.

shown in Fig. 5(a). This behavior confirms that the larger loop array dominates the radiation performance in the horizontal plane and that the smaller one contributes only a little to the cross-polarization patterns.

To explicitly show the performance enhancement offered by the two loop array combination, the HPBWs and XPDs of the three different cases shown in Fig. 5 are compared in Fig. 6 . Note that the XPDs plotted here are the minimum values within the $-60^{\circ} \leq \theta \leq+60^{\circ}$ region in the horizontal plane. As shown in the figure, the XPD can be maintained $>10 \mathrm{~dB}$ only when the two loop arrays are working together. The XPDs of the other two cases both decrease when the frequency $f \geq 2.1$ GHz. When only the large loop array is excited, the XPD is $\leq$ $10 \mathrm{~dB}$ across the band from 2.3 to $2.7 \mathrm{GHz}$, which occupies $40 \%$ of the entire operational band. When only the small loop array is excited, the results seem to be acceptable since the $\mathrm{XPD}$ is $\leq 10 \mathrm{~dB}$ only in a small percentage of the operational band, i.e., in the interval $2.55 \leq f \leq 2.7$. Moreover, the XPD is quite high for the of the operational band. However, 


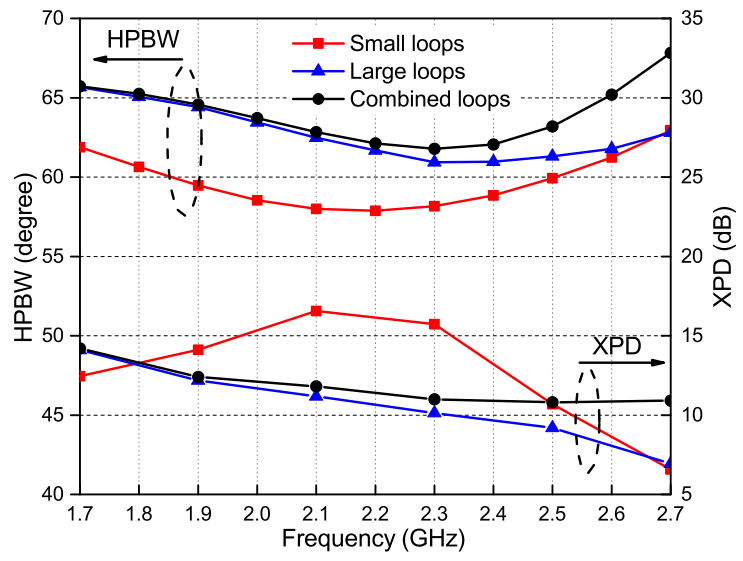

Fig. 6. Comparison of the simulated HPBW and minimum XPD values within the angular range $-60^{\circ} \leq \theta \leq+60^{\circ}$ in the horizontal plane. They were obtained from the directivity patterns generated by the rectangular loop array only, the trapezoidal loop array only, and by both arrays together.

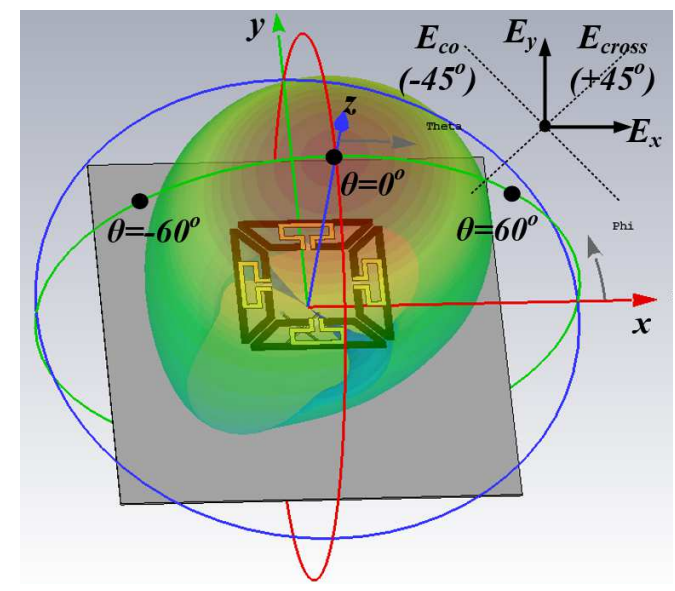

Fig. 7. Schematic 3D directivity pattern of the optimized dual-loop-array antenna.

the HPBW is quite narrow, which is unwanted. Base station antennas usually are required to have stable HPBW values of $65^{\circ} \pm 5^{\circ}$ across their entire operation frequency band [12]. Furthermore, the antenna can not be matched across the target band since the aperture is not large enough.

\section{XPD Analysis}

As suggested in [12], the XPD of this $\pm 45^{\circ}$-polarized antenna in the horizontal, $x-z$, plane was examined by decomposing the far-field shown in Fig. 7 into its $x$ - and $y$-polarized components:

$$
\widehat{x} \cdot \vec{E}=E_{x} e^{j \phi_{x}}, \widehat{y} \cdot \vec{E}=E_{y} e^{j \phi_{y}}
$$

Then the co- and cross-polarized field vectors can be expressed as follows:

$$
\begin{aligned}
E_{\text {co }} & =\cos \frac{\pi}{4} E_{x} e^{j \phi_{x}}+\cos \frac{\pi}{4} E_{y} e^{j \phi_{y}}, \\
E_{\text {cross }} & =\cos \frac{\pi}{4} E_{x} e^{j \phi_{x}}-\cos \frac{\pi}{4} E_{y} e^{j \phi_{y}} .
\end{aligned}
$$

The XPD is the ratio of the magnitudes of these co- and crosspolarized vectors:

$$
X P D=\left|\frac{E_{\text {co }}}{E_{\text {cross }}}\right|=\sqrt{\frac{E_{x}^{2}+E_{y}^{2}+2 E_{x} E_{y} \cos \left(\phi_{x}-\phi_{y}\right)}{E_{x}^{2}+E_{y}^{2}-2 E_{x} E_{y} \cos \left(\phi_{x}-\phi_{y}\right)} .}
$$

It is assumed that $\phi_{x}=\phi_{y}$ in [12] to simplify the analysis, since both the $E_{x}$ and $E_{y}$ components arrive at approximately the same time when one observes the field at a point infinitely far away. Then Eq. 3 can be rewritten as:

$$
X P D=\sqrt{\frac{E_{x}^{2}+E_{y}^{2}+2 E_{x} E_{y}}{E_{x}^{2}+E_{y}^{2}-2 E_{x} E_{y}}}=\left|\frac{E_{x}+E_{y}}{E_{x}-E_{y}}\right| .
$$

It is thus concluded that a high XPD is achieved if the difference between the magnitudes of the radiated $x$ - and $y$-polarized field components, $\left|E_{x}\right|$ and $\left|E_{y}\right|$, is as small as possible.

To have a deeper understanding of how the high XPD value is attained, Fig. 8 compares the $x$ - and $y$-polarized directivity patterns generated by the rectangular loop array, by the trapezoidal loop array, and by both two arrays together. Notice that the discrepancy between the $E_{x}$ and $E_{y}$ values is very small at $\theta=0^{\circ}$ but gets much larger as observation direction approaches $\theta= \pm 60^{\circ}$ for all the three cases. This explains why the XPD values at the edges of the target angular region in the horizontal plane are much smaller than those at boresight. In particular, as shown in Fig. 8(a), the $x$-polarized patterns are narrower than the $y$-polarized ones when only the smaller rectangular loop array is excited. Although it is difficult to discern in the figure, the $x$ and $y$-polarized patterns are getting narrower and wider, respectively, as the frequency increases. To be specific, at $\theta= \pm 60^{\circ}$, the discrepancy between the $E_{x}$ and $E_{y}$ component values increases from $E_{x} / E_{y}=-5.7$ $\mathrm{dBi}+5.0 \mathrm{dBi}=-0.7 \mathrm{~dB}$ to $E_{x} / E_{y}=-10.0 \mathrm{dBi}+2.7 \mathrm{dBi}=$ $-7.3 \mathrm{~dB}$ as the frequency increases from 1.7 to $2.7 \mathrm{GHz}$.

In contrast, Fig. 8(b) demonstrates that the $x$-polarized patterns are wider than the $y$-polarized ones when only the larger trapezoidal array is excited. Moreover, the $x$-polarized patterns are quite stable and the $y$-polarized patterns are becoming narrower as the frequency increases. Consequently, the XPD in this case is also getting smaller in agreement with the results shown in Fig. 5(b).

As shown in Fig. 8(c), the $x$ - and $y$-polarized directivity patterns radiated by the loop array combination are quite similar to those of a single trapezoidal loop array. Although $E_{x} / E_{y}$ shown in Fig. 8(c) is slightly smaller than that in Fig. 8 (b), the reduction is not significant enough to produce a large XPD enhancement according to Eq. 4. It reduces $5 \mathrm{~dB}$ at 2.7 $\mathrm{GHz}$ as shown in Fig. 6. Consequently, there must still be another factor that contributes to the XPD enhancement.

We found in our studies that while the addition of the smaller rectangular loop array to the larger trapezoidal loop array only slightly changes the magnitudes of the $E_{x}$ and $E_{y}$ components, it impacts their phases. Therefore, it was determined that it is inappropriate to assume their phases are equal, i.e, that $\phi_{x}=\phi_{y}$. Fig. 9 plots $\phi_{x}$ and $\phi_{y}$ using solid and dashed lines, respectively, for the three different array cases at $2.7 \mathrm{GHz}$. The simulated phase values are illustrated in 


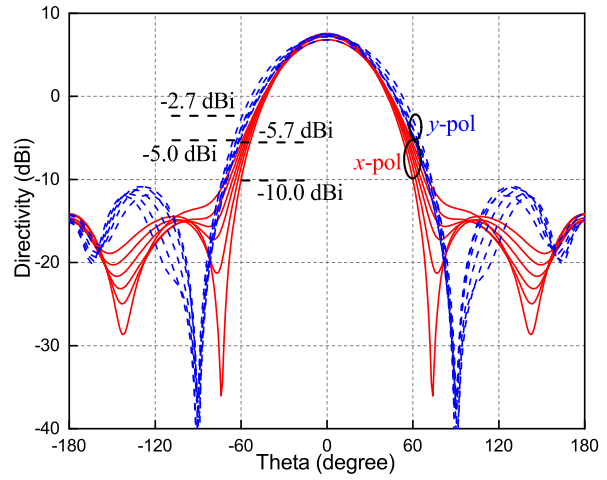

(a)

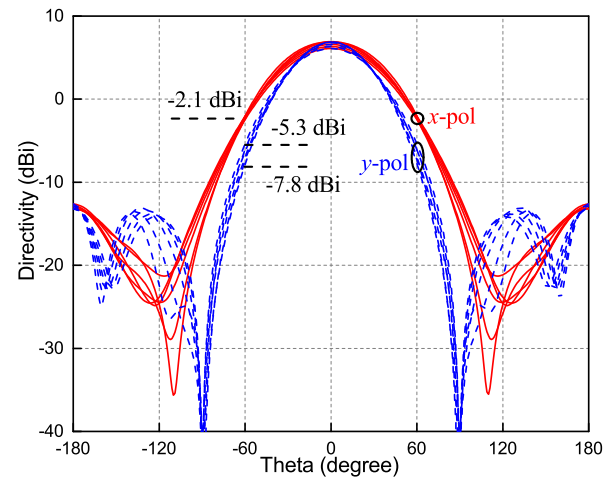

(b)

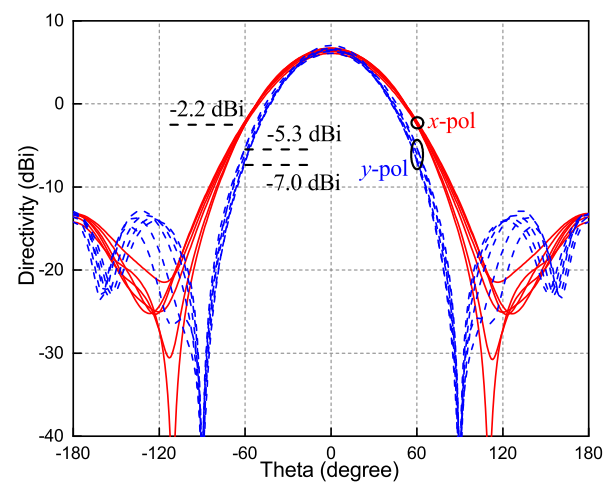

(c)

Fig. 8. Simulated $\mathrm{x}$ - and $\mathrm{y}$-polarized directivity patterns in the horizontal plane at the frequency points: $1.7,1.9,2.1,2.3,2.5$, and $2.7 \mathrm{GHz}$. They are represented by the solid and the dashed lines, respectively. (a) Rectangular loop array. (b) Trapazoidal loop array. (c) Dual-loop array.

black, red, and blue color when, respectively, only the smaller rectangular loop array, only the larger trapezoidal loop array, or both loop arrays are excited. Notice that the phase differences: $\Delta \phi=\left|\phi_{x}-\phi_{y}\right|$, for all the three cases are near zero at $\theta=0^{\circ}$ and increase with $|\theta|$. According to Eq. 3, it can then be deducted that the smaller the $\Delta \phi$, the higher the XPD value. Therefore, although the directivity pattern is observed to be dominated by the larger trapezoidal loop array contributions, the presence of the smaller rectangular loop array reduces the phase difference from $\Delta \phi_{2}=43^{\circ}$ to $\Delta \phi_{3}=26^{\circ}$ at $\theta= \pm 60^{\circ}$, thereby leading to the observed higher XPD values when the dual-loop array is excited.

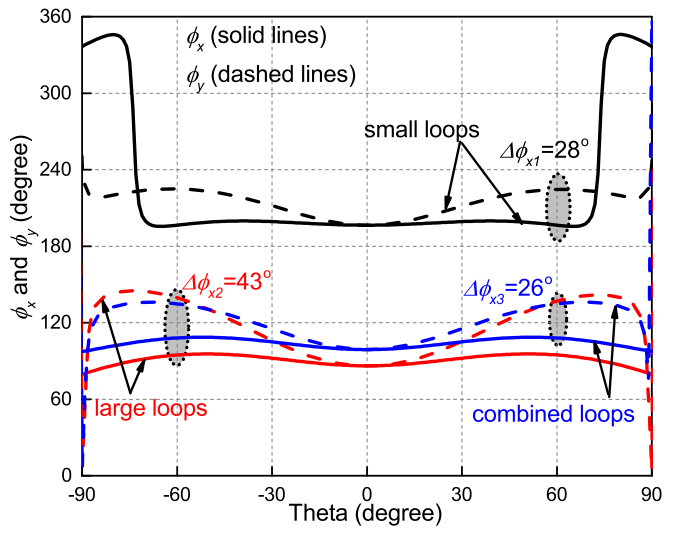

Fig. 9. Simulated phases of the $\mathrm{x}$ - and $\mathrm{y}$-polarized radiated fields in the horizontal plane at $2.7 \mathrm{GHz}$ for the rectangular loop array only, the trapezoidal loop array only, and the dual-loop array. These values are represented by the solid and the dashed lines, respectively.

\section{Antenna Design}

\section{A. Parametric Study}

The previous analysis demonstrated why high XPD values are realized across a wide band when both loop arrays are operating in concert. Further investigations were necessary to learn how to adjust the design dimensions to optimize its performance characteristics. Therefore, more parameter sweeps were conducted. Because the dual-loop array antenna configuration has many adjustable parameters, only three representative dimensions, $L_{t}, L_{r}$, and $D_{t}$, will be discussed. By changing them, the size of the trapezoidal loops, the size of the rectangular loops, and the distance between the trapezoidal loops (and, hence the size of the aperture) are changed, respectively. The performance factors concerned in these studies were the XPD, HPBW, and the input impedance. Because the feed networks are not yet included, the input impedance values are determined at the excitation ports, as shown in Fig. 3(a).

In particular, when $L_{t 2}$ was varied, all of the other parameters were fixed except for $L_{t}$. This sweep was performed with the base angles of the trapezoid being maintained and, hence, $L_{t 1}$ also had to be varied. Similar adjustments had to occur with the other parameter variations. For instance, adjusting $D_{t}$, the separation distance between the trapezoidal array elements, $D_{r}$ had to be varied appropriately to maintain the relative positions and sizes of the rectangular loops. Note that the size of the entire aperture changes when $D_{t}$ is varied, and the area of the rectangular loops change when $L_{r}$ is varied. The effects of these parameter variations on the directivity patterns and the input impedance are illustrated in Fig. 10.

As observed in Fig. 10(a), changing the size of the rectangular loops almost has no effect on the HPBW and XPD values. This outcome is mainly due to the fact that the directivity patterns are dominated by the currents on the larger trapezoidal loops. Fig. 10(b) demonstrates that the effect of the trapezoidal loop size on the radiation performance is also not significant. This outcome illustrates the robustness of this configuration. On the other hand, Fig. 10(a) and 10(b) shows that changes in the sizes of the two different loop types lead to noticeably 


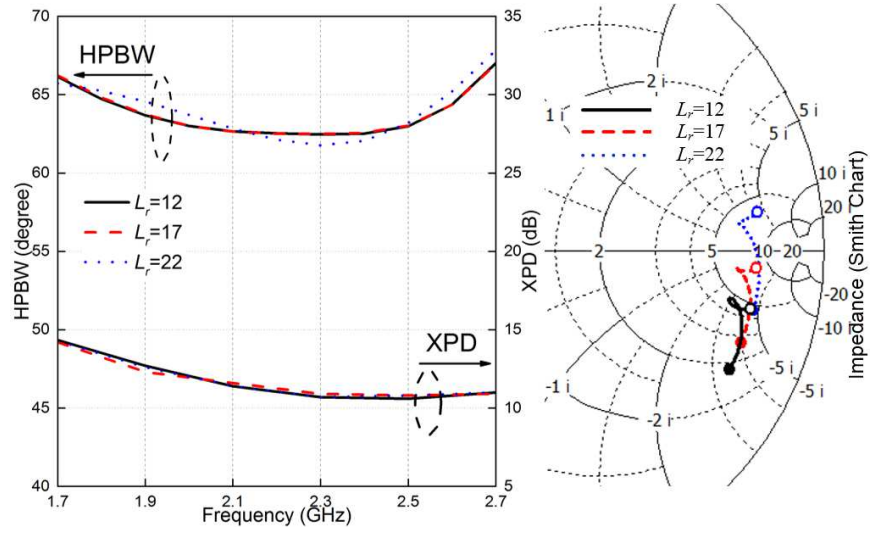

(a)

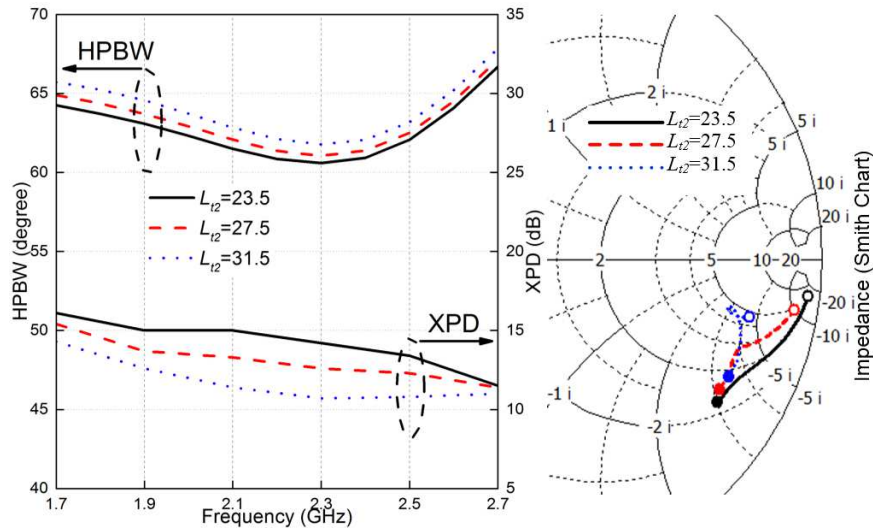

(b)

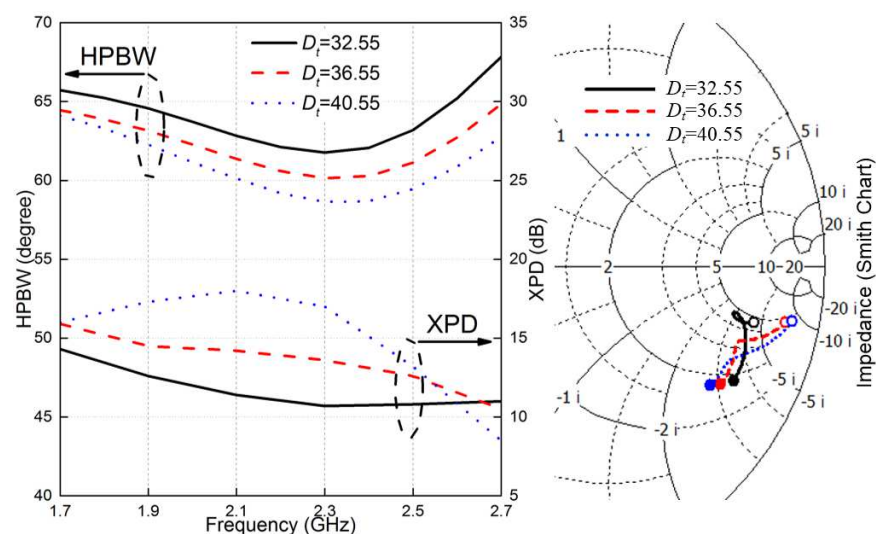

(c)

Fig. 10. Parameter sweep results given as a plot of the HPBW and XPD values in the horizontal plane as functions of the source frequency and as a Smith chart plot of the input impedance values. (a) $L_{r}$, rectangular loop size (b) $L_{t 2}$, trapezoidal look size. (c) $D_{t}$, the distance between loop radiators.

different input impedance values. These results lead to the conclusion that the length of the loops can be used effectively to tune the impedance match without having to worry about a significant change in the radiation performance.

Furthermore, as Fig. 10(c) indicates, the radiation performance is sensitive to the separation distance $D_{t}$ between the array elements. This is expected because the aperture size is a key factor in the shape of the directivity pattern. Meanwhile, $D_{t}$ also has some effects on the input impedance. It impacts the mutual coupling levels between adjacent loops. In addition,

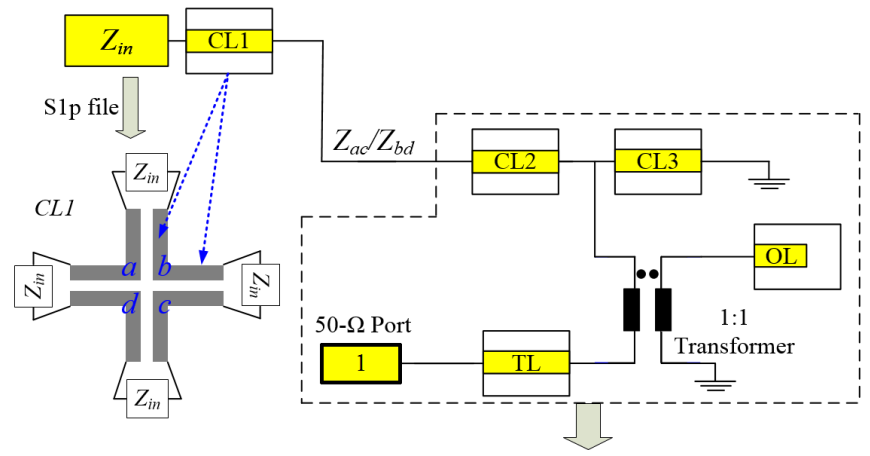

(a)

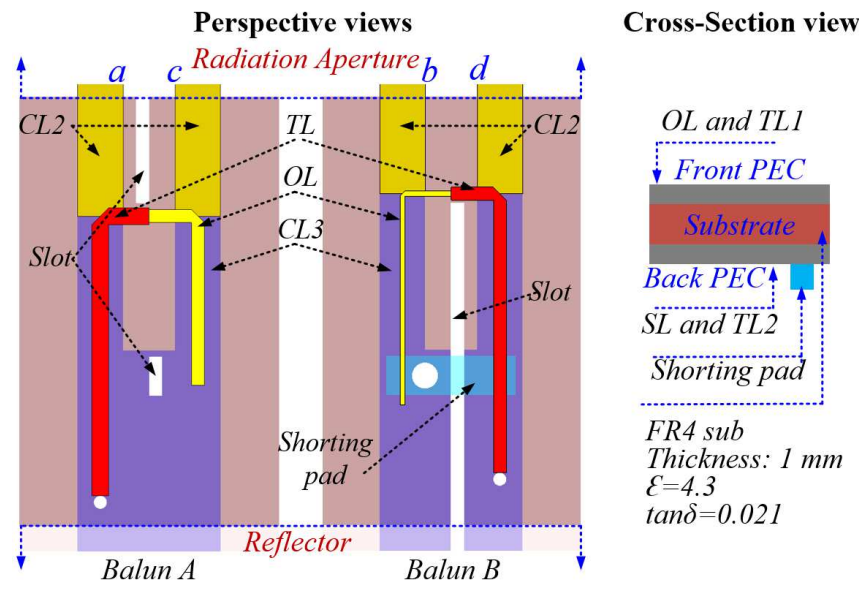

(b)

Fig. 11. Feed Networks. (a) Circuit theory model. (b) Physical implementation.

the antenna height $h$ and the ground plane size $G$ are also key factors in determining the directivity pattern and, hence, in optimizing the HPBW and XPD values. However, they have only a minor effect on the input impedance. Because they have been studied and reported previously [12], details of the effects on the performance of base station antennas caused by variations in the design parameters $h$ and $G$ will not be repeated here.

\section{B. Impedance Matching}

It was found that to optimize dual-loop antenna performance, it was best to first consider the antenna height $h$, the reflector size $G$, and the antenna aperture size. The length and width of the loops were then be adjusted to achieve a satisfactory input impedance match across the target frequency band. To design the feed network, the input impedances, $Z_{i n}$, at the excitation ports shown in Fig. 3(a) were exported from the full wave simulator as a S1p file and imported into the matching circuit model shown in Fig. 11(a). By optimizing this matching circuit with circuit simulations, which are faster and more cost-effective than those in a full-wave simulator, it was determined that the antenna could be matched to the source. Once the optimized circuit parameters were attained, a 3D model of the feed network was then built and adjusted with full-wave simulations. The final design is shown in Fig. 11(b). The matching circuit consists of two major parts. The 
TABLE II

DIMENSIONS OF THE TWO FEED NETWORKS (IN MILLIMETRES)

\begin{tabular}{|c|c|c|c|c|c|c|}
\hline-- & \multicolumn{3}{|c|}{ Feed network A } & \multicolumn{3}{c|}{ Feed network B } \\
\hline (mm) & Length & Width & Distance & Length & Width & Distance \\
\hline CL1 & 15.22 & 3.0 & 2.16 & 15.22 & 3.0 & 2.16 \\
CL2 & 8.5 & 3.5 & 4.0 & 8.5 & 3.5 & 4.0 \\
CL3 & 10.32 & 3.5 & 4.0 & 12.02 & 3.5 & 4.0 \\
TL & 23.95 & 1.3 & -- & 24.25 & 1.0 & -- \\
OL & 15.75 & 1.0 & -- & 19.65 & 0.4 & -- \\
\hline
\end{tabular}

first part is four pairs of coupled lines (CL1) printed on the same surface as the loop radiators. As shown in Figs. 11(a) and 2(a), the trace CL1 links all of the loop radiators together. Therefore, by placing a differential voltage across $a c$ or $b d$, all of the loop radiators can be excited to achieve the desired $\pm 45^{\circ}$ dual-polarization.

This filter type of impedance matching feed network was first proposed in [27] and then used in [12,13] for base station antennas. The principle is to utilize several segments of transmission lines together with the impedance of the antenna itself to construct both a band-pass filter and a resistance transformer to mitigate, respectively, the variations of the reactance and resistance of the input impedance. The matching components and their arrangement are not fixed. They can be adjusted subject to the geometrical constraints placed on the system.

The other part of the feed network illustrated in Fig. 11(b) is a set of baluns. They are oriented perpendicular to the radiation aperture and the reflector. This arrangement transfers $Z_{a c} / Z_{b d}$ into $50-\Omega$. It provides geometrical support to the radiators. These baluns are composed of four components: two pairs of coupled lines, CL2 and CL3; an open circuit line, OL; and a transmission line, TL. As shown in Fig. 11(b), CL2 and CL3 are printed on the back side of the substrate and are shorted to the ground (reflector). They work as the back (ground plane) metal for the microstrip lines TL and OL that are printed on the front side of the substrate. The 1:1 transformer shown in Fig. 11(a) represents the coupling between these microstrip lines and the coupled lines printed on the front and back sides of the substrate, respectively.

Slots are etched into the two feed networks for assemblage. To avoid overlap, these two feed networks are designed to be slightly different. In particular, the length of CL2 in each has a different value. This feature subsequently leads to different dimension values for the components CL3, OL, and TL for the two feed networks to retune the impedance back to 50 $\Omega$. Table II gives the dimensions of these two feed network designs. Note that the TL and OL are all bent towards the middle. Their lengths in Table II are the total lengths of the two segments.

\section{RESUlts}

The optimized dual-loop antenna was fabricated and measured. Fig. 12 is a photograph of the fabricated prototype. Note that one can see the trapezoidal loops printed on the back side because the substrate is semi-transparent. The Sparameters of this antenna were measured in the RF lab of the

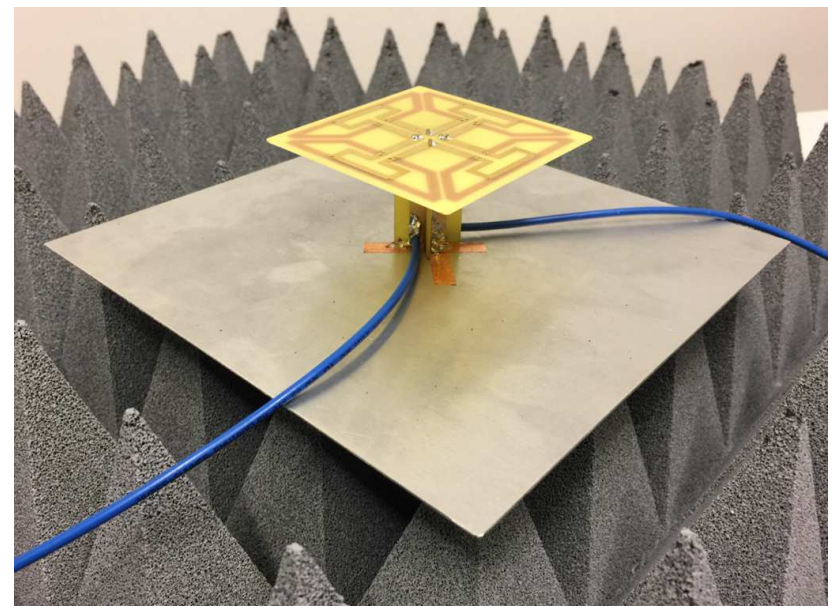

Fig. 12. Photo of the fabricated antenna prototype.

Global Big Data Technologies Centre (GBDTC), University of Technology Sydney (UTS), Sydney, Australia. Figs. 13(a) and 13(b) show the simulated and measured reflection coefficients and transmission coefficients of the two ports, respectively. It is observed that the measured results agree quite well with their simulated values. Across the target band from 1.7 to 2.7 $\mathrm{GHz}$, the magnitudes of the measured reflection coefficients of the two ports, $\left|S_{11}\right|$ and $\left|S_{22}\right|$, are $<-10 \mathrm{~dB}$ and those of the measured transmission coefficients, $\left|S_{12}\right|$ and $\left|S_{21}\right|$, are $<-23$ $\mathrm{dB}$. Thus, the antenna has a $1.0 \mathrm{GHz}$ bandwidth and, hence, a FBW $=45.5 \%$. Note that only the $\left|S_{21}\right|$ results are given in Fig. 13(b) since the $\left|S_{12}\right|$ results are essentially the same.

The radiation performance of the dual-loop antenna prototype was measured at the outdoor antenna range owned by Vecta Pty Ltd [28], located in Castle Hill, Sydney, Australia. The measured and simulated co- and cross-polarization directivity patterns in the horizontal plane are shown in Cartesian form in Fig. 14. Four frequency samples were selected within the operational band, 1.7 to $2.7 \mathrm{GHz}$. As illustrated in the figure, nearly identical patterns were achieved across the band; and the measured results agree very well with the simulated ones. Fig. 15 gives the simulated and measured HPBWs in the horizontal plane of the dual-polarization directivity patterns. It confirms that stable radiation patterns were attained, their HPBWs varying from $63^{\circ}$ to $70^{\circ}$ for both polarizations. The measured XPD results are illustrated in Fig. 16. It can be seen that the measured XPDs are $>20 \mathrm{~dB}$ at boresight and are $>$ $10 \mathrm{~dB}$ within the entire $-60^{\circ} \leq \theta \leq 60^{\circ}$ angular range.

\section{CONCLUSION}

A novel dual-loop array antenna for base station applications was reported that has a wide band and is dual-polarized with enhanced XPD values. The design consists of two loop arrays, one with four rectangular loops and one with four trapezoidal loops, printed on two sides of a substrate. The larger trapezoidal loops behave like folded dipoles having an electric current character. The smaller rectangular loops support loop currents and, hence, act primarily as loop radiators acting as magnetic dipoles. Comparisons were made between the radiation performance attained by exciting only the smaller 


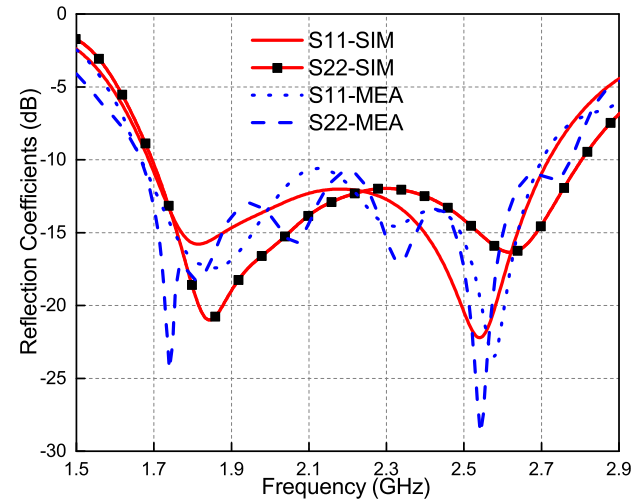

(a)

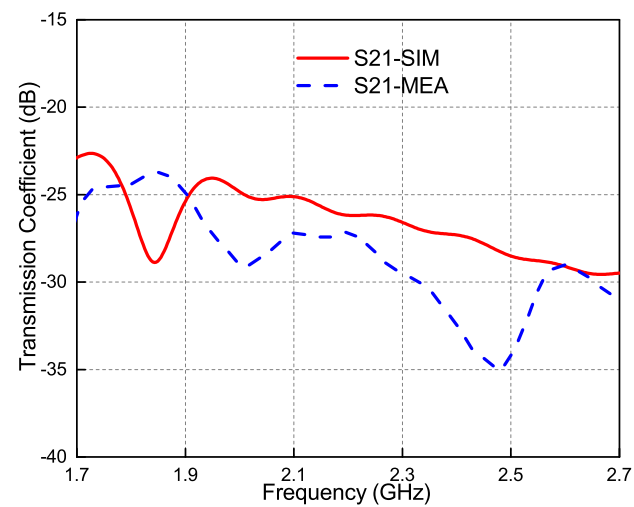

(b)

Fig. 13. Simulated and measured S-parameters of the dual-loop array antenna prototype. (a) Reflection coefficients. (b) Transmission coefficients.

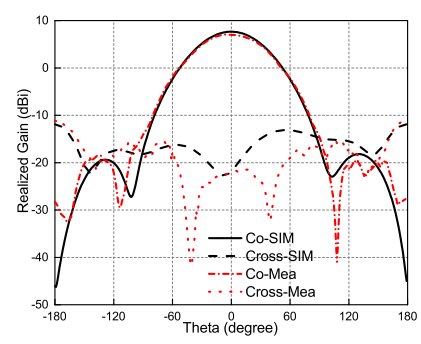

(a)

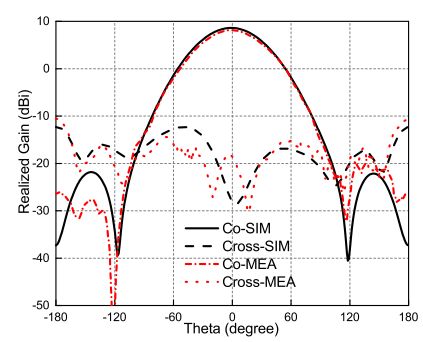

(c)

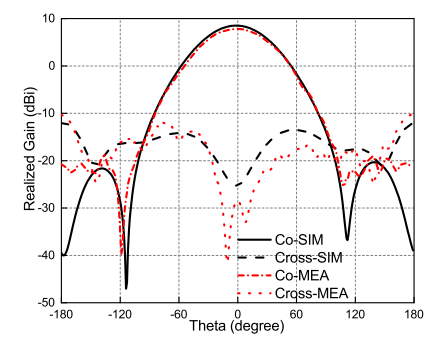

(b)

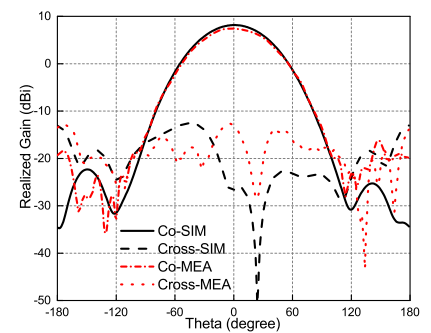

(d)
Fig. 14. Simulated and measured co- and cross-polarization directivity patterns of the dual-loop antenna prototype in its horizontal plane at (a) 1.7, (b) 2.0 , (c) 2.4. and (d) $2.7 \mathrm{GHz}$.

rectangular array and only the larger trapezoidal loop array, as well as the two array combination to demonstrate the superior performance characteristics of the dual-loop array configuration. Current distributions on the radiators were presented

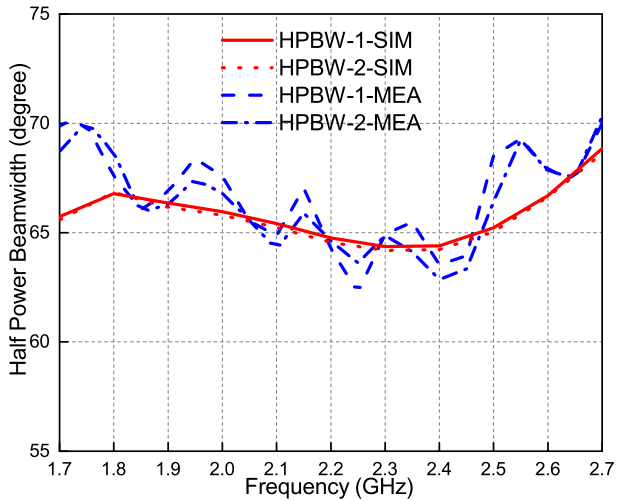

Fig. 15. Simulated and measured HPBWs of the dual-loop antenna prototype in its horizontal plane.

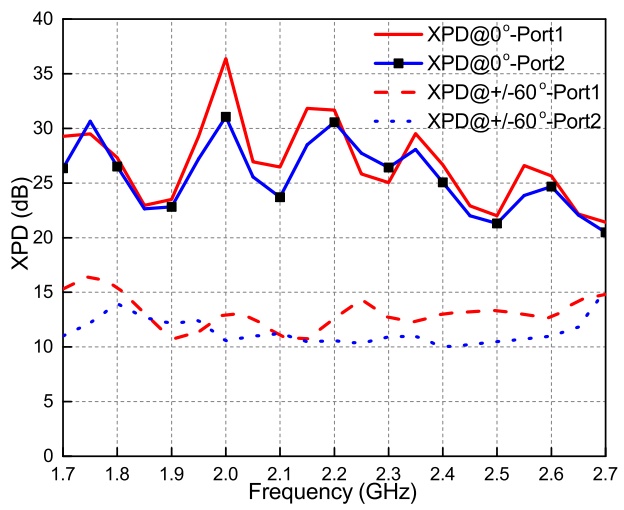

Fig. 16. Measured XPD values of the dual-loop antenna prototype at boresight $\left(\theta=0^{\circ}\right)$ and at the edges of its angular coverage, $\left.\theta= \pm 60^{\circ}\right)$.

along with a theoretical analysis to explain the origin of its enhanced XPD values. Parameter studies were discussed to show the robustness of this configuration. The antenna's feed networks were shown to be designed to excite the two arrays simultaneously with good impedance matching.

A prototype dual-loop antenna was fabricated and tested. The measured results, in good agreement with their simulated values, demonstrate that across its operational band from 1.7 to $2.7 \mathrm{GHz}(100 \mathrm{MHz} \mathrm{BW}$ and $\mathrm{FBW}=45.5 \%)$, the $S_{11}$ and $S_{22}$ values of its two ports are $<-10 \mathrm{~dB}$, and its $S_{12}$ and $S_{21}$ values are $<-23 \mathrm{~dB}$. The measured HPBWs are $66.5^{\circ} \pm 3.5^{\circ}$, the XPD values at $\theta=0^{\circ}$ are $>20 \mathrm{~dB}$, and the minimum XPD values within the $-60^{\circ} \leq \theta \leq 60^{\circ}$ angular range are $>$ $10 \mathrm{~dB}$. The attained XPD values, impedance match, isolation, and stable radiation patterns make the reported dual-loop array antenna attractive for base station applications. Moreover, the approach of adding additional magnetic radiators to improve the XPD values established herein could also be applied to other dual-polarized antenna designs.

\section{REFERENCES}

[1] H. D. Hristov, H. Carrasco, R. Feick, and B. L. Ooi, "Low-profile $\mathrm{X}$ antenna with flat reflector for polarization diversity applications," Microwave and Optical Technology Letters, vol. 51, pp. 1508-1512, 2009.

[2] H. D. Hristov, H. Carrasco, R. Feick, and G. S. Kirov, "Broadband two-port $\mathrm{X}$ antenna array with flat reflector for polarization diversity 
applications," Microwave and Optical Technology Letters, vol. 52, pp. 2833-2837, Dec. 2010.

[3] Y. Liu, H. Li, F. W. Wang, and S. X. Gong, "A Novel Miniaturized Broadband Dual-Polarized Dipole Antenna for Base Station," Microwave and Optical Technology Letters, vol. 12, pp. 1335-1338, Oct. 2013.

[4] Y. Gou, S. Yang, J. Li and Z. Nie, "A Compact Dual-Polarized Printed Dipole Antenna With High Isolation for Wideband Base Station Applications," IEEE Transactions on Antennas and Propagation, vol. 62, no. 8, pp. 4392-4395, Aug. 2014.

[5] Z. Bao, Z. Nie and X. Zong, "A Novel Broadband Dual-Polarization Antenna Utilizing Strong Mutual Coupling," IEEE Transactions on Antennas and Propagation, vol. 62, no. 1, pp. 450-454, Jan. 2014.

[6] Y. Cui, R. Li and H. Fu, "A Broadband Dual-Polarized Planar Antenna for 2G/3G/LTE Base Stations," IEEE Transactions on Antennas and Propagation, vol. 62, no. 9, pp. 4836-4840, Sept. 2014.

[7] Q. X. Chu, D. L. Wen and Y. Luo, "A Broadband $\pm 45^{\circ}$ Dual-Polarized Antenna With Y-Shaped Feeding Lines," IEEE Transactions on Antennas and Propagation, vol. 63, no. 2, pp. 483-490, Feb. 2015.

[8] S. X. Ta, I. Park, and R. W. Ziolkowski, "Crossed Dipole Antennas: A review," IEEE Antennas and Propagation Magazine, vol. 57, no. 5, pp. 107-122, Oct. 2015.

[9] B. B. Jones and J. K. A. Allan, "Ultra-Wideband Dual-Band Cellular Base Station Antenna," US Patent 2014/0139387 A1, May, 2014.

[10] H. Huang, Y. Liu, and S. X. Gong, "A Broadband Dual-Polarized Base Station Antenna With Anti-Interference Capability," IEEE Antennas and Propagation Letters, vol. 16, pp. 613-616, Mar. 2017.

[11] D. Z. Zheng and Q. X Chu, "A Multimode Wideband $\pm 45^{\circ}$ DualPolarized Antenna With Embedded Loops," IEEE Antennas and Propagation Letters, vol. 16, pp. 613-616, Mar. 2017.

[12] C. Ding, H. H. Sun, R. W. Ziolkowski, and Y. J. Guo, "Simplified Tightly-Coupled Cross-Dipole Arrangement for Base Station Applications," IEEE Access, vol. 5, pp. 27491-27503, 2017.

[13] H. H. Sun, C. Ding, B. Jones, and Y. J. Guo, "A Wideband Base Station Antenna Element with Stable Radiation Pattern and Reduced Beam Squint," IEEE Access, vol. 5, pp. 23022-23031, 2017.

[14] B. Q. Wu and K. M. Luk "A Broadband Dual-Polarized MagnetoElectric Dipole Antenna With Simple Feeds," IEEE Antennas and Wireless Propagation Letters, vol. 8, pp. 60-63, 2009.

[15] K. M. Luk and B. Wu, "The Magnetoelectric Dipole - A Wideband Antenna for Base Stations in Mobile Communications," Proceedings of the IEEE, vol. 100, no. 7, pp. 2297-2307, July 2012.

[16] H. Zhai, J. Zhang, Y. Zang, Q. Gao and C. Liang, "An LTE Base-Station Magnetoelectric Dipole Antenna with Anti-Interference Characteristics and Its MIMO System Application," IEEE Antennas and Wireless Propagation Letters, vol. 14, pp. 906-909, 2015.

[17] G. Idayachandran and R. Nakkeeran, "Compact magneto-electric dipole antenna for LTE femtocell base stations," Electronics Letters, vol. 52, no. 8, pp. 574-576, Apr. 2016.

[18] M. Li, K. M. Luk, L. Ge and K. Zhang, "Miniaturization of Magnetoelectric Dipole Antenna by Using Metamaterial Loading," IEEE Transactions on Antennas and Propagation, vol. 64, no. 11, pp. 49144918, Nov. 2016.

[19] S. G. Zhou, Z. H. Peng, G. L. Huang and C. Y. D. Sim, "Design of a Novel Wideband and Dual-Polarized MagnetoElectric Dipole Antenna," IEEE Transactions on Antennas and Propagation, vol. 65, no. 5, pp. 2645-2649, May 2017.

[20] C. D. Balanis, Antenna Theory: Analysis and Design. 4th Ed., New York, NY, USA: John Wiley \& Sons, 2015.

[21] Y. H. Huang, Q. Wu and Q. Z. Liu, "Broadband dual-polarised antenna with high isolation for wireless communication," Electronics Letters, vol. 45, no. 14, pp. 714-715, Jul. 2009.

[22] S. G. Zhou, P. K. Tan and T. H. Chio, "Low-Profile, Wideband DualPolarized Antenna With High Isolation and Low Cross Polarization," IEEE Antennas and Wireless Propagation Letters, vol. 11, pp. 1032-1035, 2012.

[23] H. Huang, Y. Liu, and S. X. Gong, "A Novel Dual-Broadband, DualPolarized Antenna for 2G/3G/LTE Base Stations," IEEE Transactions on Antennas and Propagation, Vol. 64, No.9, pp. 4113-4118, 2016.

[24] H. H. Sun, C. Ding, T. S. Bird, and Y. J. Guo, "A Base Station Antenna Element with Simple Structure but Excellent Performance," in $3^{\text {rd }}$ Australian Microwave Symposium, Feb, 2018, Brisbane, Australia.

[25] Y. Luo, Q. X. Chu and D. L. Wen, "A Plus/Minus 45 Degree Dual-Polarized Base-Station Antenna With Enhanced Cross-Polarization Discrimination via Addition of Four Parasitic Elements Placed in a Square Contour," IEEE Transactions on Antennas and Propagation, vol. 64, no. 4, pp. 1514-1519, April 2016.
[26] Y. Luo, Q. X. Chu and J. Bornemann, "Enhancing cross-polarisation discrimination or axial ratio beamwidth of diagonally dual or circularly polarised base station antennas by using vertical parasitic elements," IET Microwaves, Antennas and Propagation, vol. 11, no. 9, pp. 1190-1196, Jul. 2017.

[27] C. Ding, B. Jones, Y. J. Guo and P.-Y. Qin, "Wideband Matching of FullWavelength Dipole With Reflector for Base Station," IEEE Transactions on Antennas and Propagation, vol. 65, no. 10, Oct. 2017.

[28] VectaLabs. Accessed: Oct. 25, 2017. [Online]. Available: http://vectalabs.com 